\title{
Evaluating the costs and benefits of using combination therapies
}

\section{Fixed-dose combination therapy can improve compliance; but at what cost?}

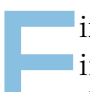

ixed-dose combination (FDC) therapies, which involve combining two or more pharmaceutical drugs in a single tablet, are being increasingly prescribed in Australia, particularly for people with long-term chronic conditions such as diabetes and cardiovascular disease. The use of combination therapies can reduce out-of-pocket costs to the patient (ie, there is only one dispensing fee of $\$ 6.60$ and copayment of up to $\$ 36.90)$. There is evidence that patients given combination therapies have greater adherence and compliance than those taking these medications separately. ${ }^{1}$ However, combination therapies that are subsidised through Australia's Pharmaceutical Benefits Scheme (PBS) can be more costly than using the component therapies. Our aim is to review evidence on benefits, use and the costs of combination therapies. We then propose a framework for pricing and evaluation of combinations that would ensure they are a cost-effective option for the Australian health care system.

The growth in use of combination drugs to treat diabetes and cardiovascular disease is illustrated in Box 1, which shows total expenditure on these therapies over time. Australian guidelines for the treatment of blood pressure (BP) recognise that combinations of antihypertensive drugs are required to achieve BP targets. $^{2}$ Similarly, patients with type 2 diabetes are often on multiple therapies to adequately control their blood glucose. ${ }^{3}$ The clinical rationale for using FDCs is underpinned by studies such as the UMPIRE (Use of a Multidrug Pill in Reducing Cardiovascular Events) trial, ${ }^{4}$ which showed an improvement in adherence for patients taking FDC cardiovascular medications. An earlier metaanalysis of three cohort studies and two clinical trials of FDC antihypertensive agents showed a $21 \%$ increase in compliance compared with corresponding free-drug combinations (95\% CI, 1.03-1.43) and a non-significant trend toward a reduction in BP levels. ${ }^{1}$

Combination therapies are evaluated by the Pharmaceutical Benefits Advisory Committee (PBAC) following industry submissions, which generally justify listing a combination therapy on the PBS on the basis of cost minimisation, which means that the combination is clinically non-inferior to the separate components at the same or a lower price. ${ }^{5}$ While the initial price of a combination product is generally lower, subsequent reductions in the price of the FDC drug are not
Philip M Clarke $\mathrm{BEc}, \mathrm{MEc}, \mathrm{PhD}$ Professor

Alex B Avery $\mathrm{BSc}$ (Hons) Research Assistant

Centre for Health Policy, Melbourne School of Population and Global Health, University of Melbourne, Melbourne, VIC.

philip.clarke@ unimelb.edu.au

doi: $10.5694 / \mathrm{mjal} 4.00199$

There are several circumstances under which the combination therapy can become more expensive than the sum of the individual therapies

Online first 28/04/14 necessarily linked to equivalent reductions in the prices of components.

There are several circumstances under which the combination therapy can become more expensive than the sum of the individual therapies.

First, the PBAC must advise the Minister for Health if there is a significant improvement in compliance, a significant improvement in efficacy or a significant reduction in toxicity over the alternative therapies. ${ }^{5}$ The Minister may act on this advice when pricing combinations, so that there are smaller or no price reductions for such products. In practice, the PBAC has only rarely provided this advice, as a search of PBAC indicates that ezetimibe and simvastatin is one of the few combinations that have been deemed to fulfil these criteria. Even for this FDC, the additional cost to the government is around $\$ 20$ million per year. In contrast to the PBAC, the National Institute for Health and Clinical Excellence in the United Kingdom does not recommend using FDCs with ezetimibe due to the higher cost. ${ }^{6}$

Second, only single-brand combination items have their costs linked to their component drug therapy items, so when prices of the components fall, these reductions flow onto the FDC. When there are multiple brands of the same combination (even if they are supplied by the same manufacturer), the costs are subject to a mechanism known as price disclosure. ${ }^{7}$ This mechanism bases future PBS subsidies on the average wholesale cost to pharmacies of individual drugs, so over time the government pays a cost that reflects the market price. When multiple brands of an FDC are available, price disclosure only takes into account the wholesale costs of these drugs, and there is no link to the cost of the separate components.

The current pricing arrangements have a significant impact on the way many FDCs are priced relative to their component therapies. A prime example is the combination of clopidogrel with aspirin, which can reduce the risk of cardiovascular events, but at an increased risk of bleeding compared with aspirin alone. ${ }^{8}$ The PBAC recommended listing the combination on the PBS for the treatment of acute coronary syndromes and long-term atherothrombotic events on a costminimisation basis, and it became available in late 2009 .

Box 2 shows the cost per dose of clopidogrel compared with the FDC with aspirin. On initial PBS listing, the price of the combination was set at 1 cent cheaper than the cost of clopidogrel and this was maintained until a month before the PBS subsidy for clopidogrel was due to decline by $18 \%$ because of the price disclosure 
1 Total government expenditure on combinations by therapy class*

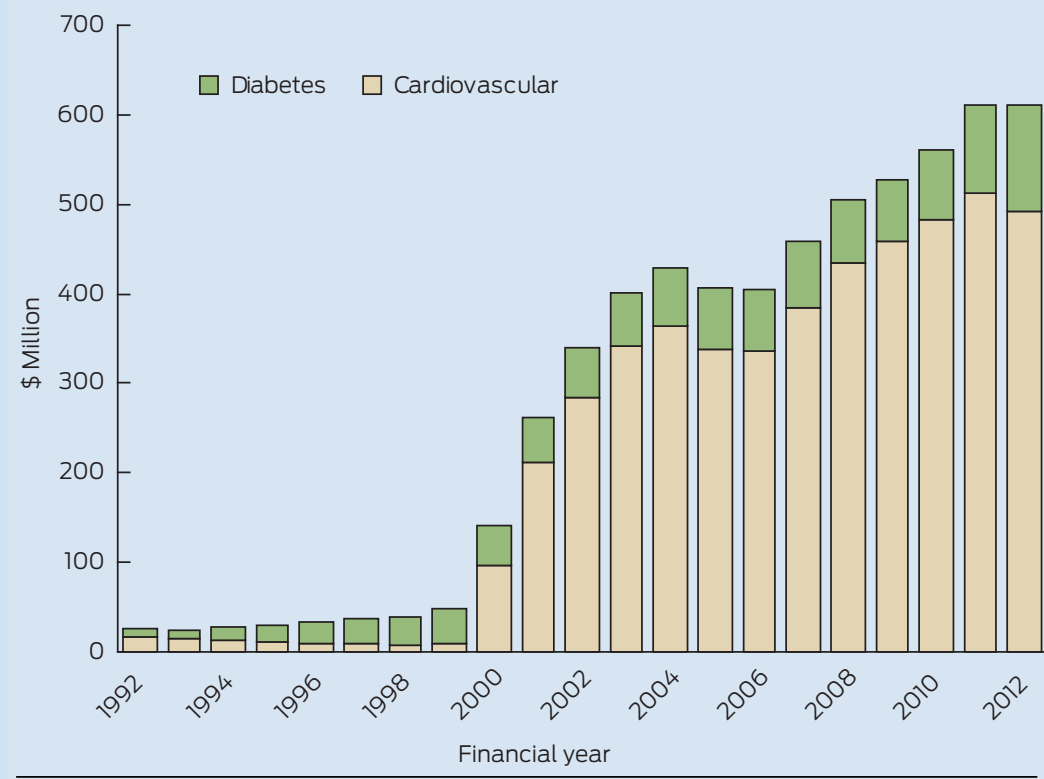

*Adjusted for inflation to 2012 dollar value using gross domestic product final consumption expenditure price deflator. drug list, thus exempting the FDC from the $25 \%$ price cut to atorvastatin in December $2012 .{ }^{9}$ The most common FDC of this therapy (10 mg amlodipine and $40 \mathrm{mg}$ atorvastatin) currently has a dispensed price of \$76.10, while the dispensed price of these purchased separately would be $\$ 40.78$ with consequently a lower cost to the government.

A new pricing framework is needed to ensure these medications are a cost-effective option for government and patients. The most obvious reform is to permanently link the dispensed price of FDCs to their individual components, rather than just for an initial period after their listing on the PBS. There is precedence for the PBAC to take this approach, as it has recommended linking the price of two off-patent drugs, atorvastatin and simvastatin, although this has yet to adopted by the government. ${ }^{9}$

A price differential in favour of FDCs could be justified if they can be shown to improve adherence in a general practice setting and thereby reduce risk factors for these chronic diseases. A key source of evidence could come from commissioning pragmatic randomised controlled trials of FDCs versus free-dose combinations in an Australian health care setting. Such studies should distinguish between the effects of charging the patient a lower out-of-pocket cost and the improved adherence associated with the convenience of having to take fewer tablets. If the results of these trials show improved clinical outcomes, then the PBAC could determine a price premium for these therapies based on the additional expected health benefits. ${ }^{10}$ Such an approach would be far superior to the current process, which involves minimal evaluation of combinations and a flawed regulatory pricing system that generates large long-term costs to the taxpayer.

The recent McKeon review into health and medical research suggests that the government invest $3 \%-4 \%$ of current health expenditure on research to improve the

\section{Cost of clopidogrel compared with clopidogrel and aspirin combination*} sub-committees, as almost all combinations have been listed on the basis of cost minimisation, even though the subsequent PBS subsidy often exceeds the costs of component therapies after pricing becomes delinked. The overall cost to the government of listing diabetes and cardiovascular combination therapies is around $\$ 120$ million more annually, compared with the sum of their equivalent individual therapies.

Further, the uptake of some combination therapies has been much greater than forecast in submissions to the PBAC. For example, the amlodipine and atorvastatin combination was forecast by the manufacturer to involve fewer than 200000 prescriptions within the first 4 years after listing on the PBS (at an estimated cost to the PBS of less than $\$ 10$ million per year). Based on Medicare Australia data, the actual volume of prescriptions over the first 4 years was 2.95 million, at a total cost of $\$ 215$ million. ${ }^{1}$ While this therapy was listed on a costminimisation basis, the link between the price of the combination and the component drugs was broken when the manufacturer introduced a second brand of the same drug. This again resulted in moving off the combination

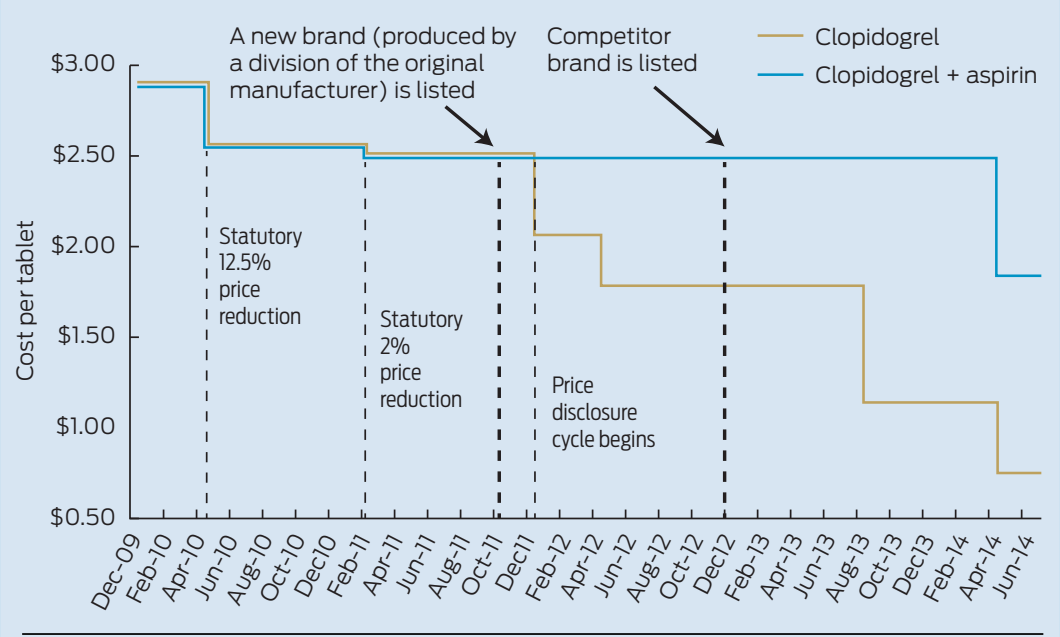

* Cost per tablet $(\$)$ pricing is calculated from dispensed prices as per the Pharmaceutical Benefits Scheme for the respective month. Prices from April 2014 are based on calculated prices from the National Health (Weighted average disclosed price - main disclosure cycle) Determination 2013 (No. 2) and Medicare Australia's "Pricing of PBS Medicine". 
health care system. ${ }^{11}$ In the context of cardiovascular and diabetes combination therapies, Australia is spending \$600 million per year, so around \$18-\$24 million annually on research would be consistent with the report's recommendations. In the current financially constrained environment, where could this money be found? If there was no price premium for combinations that have been listed on the PBS on a cost-minimisation basis, it would save around six times this amount. Given the government's responsibility to ensure an equitable and sustainable health care system, only paying a premium for combination therapies where there is demonstrated evidence of clinical benefit would reduce waste in the Australian health care system.

Competing interests: No relevant disclosures.

Provenance: Not commissioned; externally peer reviewed.

1 Gupta AK, Arshad S, Poulter NR. Compliance, safety, and effectiveness of fixed-dose combinations of antihypertensive agents: a meta-analysis. Hypertension 2010; 55: 399-407.

2 National Heart Foundation of Australia (National Blood Pressure and Vascular Disease Advisory Committee). Guide to management of hypertension 2008. Updated December 2010. http://www. heartfoundation.org.au/SiteCollectionDocuments/HypertensionGuidelines 2008to2010Update.pdf (accessed Apr 2014).
3 Raz I. Guideline approach to therapy in patients with newly diagnosed type 2 diabetes. Diabetes Care 2013; 36 Suppl 2: S139-S144.

4 Thom S, Poulter N, Field J, et al; UMPIRE Collaborative Group. Effects of a fixed-dose combination strategy on adherence and risk factors in patients with or at high risk of CVD: the UMPIRE randomized clinical trial. JAMA 2013; 310: 918-929.

5 Pharmaceutical Benefits Advisory Committee. Guidelines for preparing, submissions to the Pharmaceutical, Benefits Advisory Committee. Version 4.4. 2008. http://www.pbac.pbs.gov.au (accessed Apr 2014).

6 National Institute for Clinical Excellence. Ezetimibe for the treatment of primary (heterozygous-familial and non-familial) hypercholesterolaemia NICE technology appraisal guidance 132. London: NICE, 2007. http://www. nice.org.uk/nicemedia/live/11886/38799/38799.pdf (accessed Apr 2014).

7 Australian Government Department of Health and Ageing. Pharmaceutical Benefits Scheme expanded and accelerated price disclosure arrangements. Procedural and operational guidelines. 2010. http://www.pbs.gov.au/ industry/pricing/eapd/eapd-guidelines.pdf (accessed Apr 2014).

8 Squizzato A, KellerT, Romualdi E, Middeldorp S. Clopidogrel plus aspirin versus aspirin alone for preventing cardiovascular disease. Cochrane Database Syst Rev 2011; (1): CD005158.

9 Clarke PM. The pricing of statins and implications for Pharmaceutical Benefits Scheme expenditure. Med J Aust 2013; 198: 260.

10 Claxton K, Briggs A, Buxton MJ, et al. Value based pricing for NHS drugs: an opportunity not to be missed? BMJ 2008; 336: 251-254.

11 Australian Government Department of Health and Ageing. Strategic review of health and medical research: better health through research. Canberra: Commonwealth of Australia, 2013. http://www.mckeonreview. org.au/downloads/Strategic_Review_of_Health_and_Medical_Research_ Feb_2013-Final_Report.pdf (accessed Apr 2014). 